\title{
Le potentiel de la production forestière paysanne en Amazonie brésilienne
}

Philippe SABLAYROLles ${ }^{1}$ Hildemberg CRUZ ${ }^{2}$

Marcelo SANTOS MELO 3 Isabel GARCIA DRIGO4 Plínio SIST ${ }^{5}$

\section{${ }^{1}$ Gret}

Rua Antônio Barreto 983

Ap. 1301, Umarizal, 66055-050

Belém-PA

Brésil

2 IBAMA, SUPES, IBAMA/PA

Avenida Conselheiro Furtado, 1303

Batista Campos, 66.000-000

Belém-PA

Brésil

${ }^{3}$ SFB

Unidade Avançada da BR 163

Rua Rosa Vermelha, 739

Aeroporto Velho, 68.020-100

Santarém-PA

Brésil

4 Universidade São Paulo, NESA/USP

Departamento de Economia

Sala C 112 do prédio FEA I

Av. Prof. Luciano Gualberto, 908

Cidade Universitária, 05508-900

São Paulo-SP

Brésil

${ }^{5}$ Cirad

UR B\&SEF

Campus international de Baillarguet 34398 Montpellier Cedex 5

France

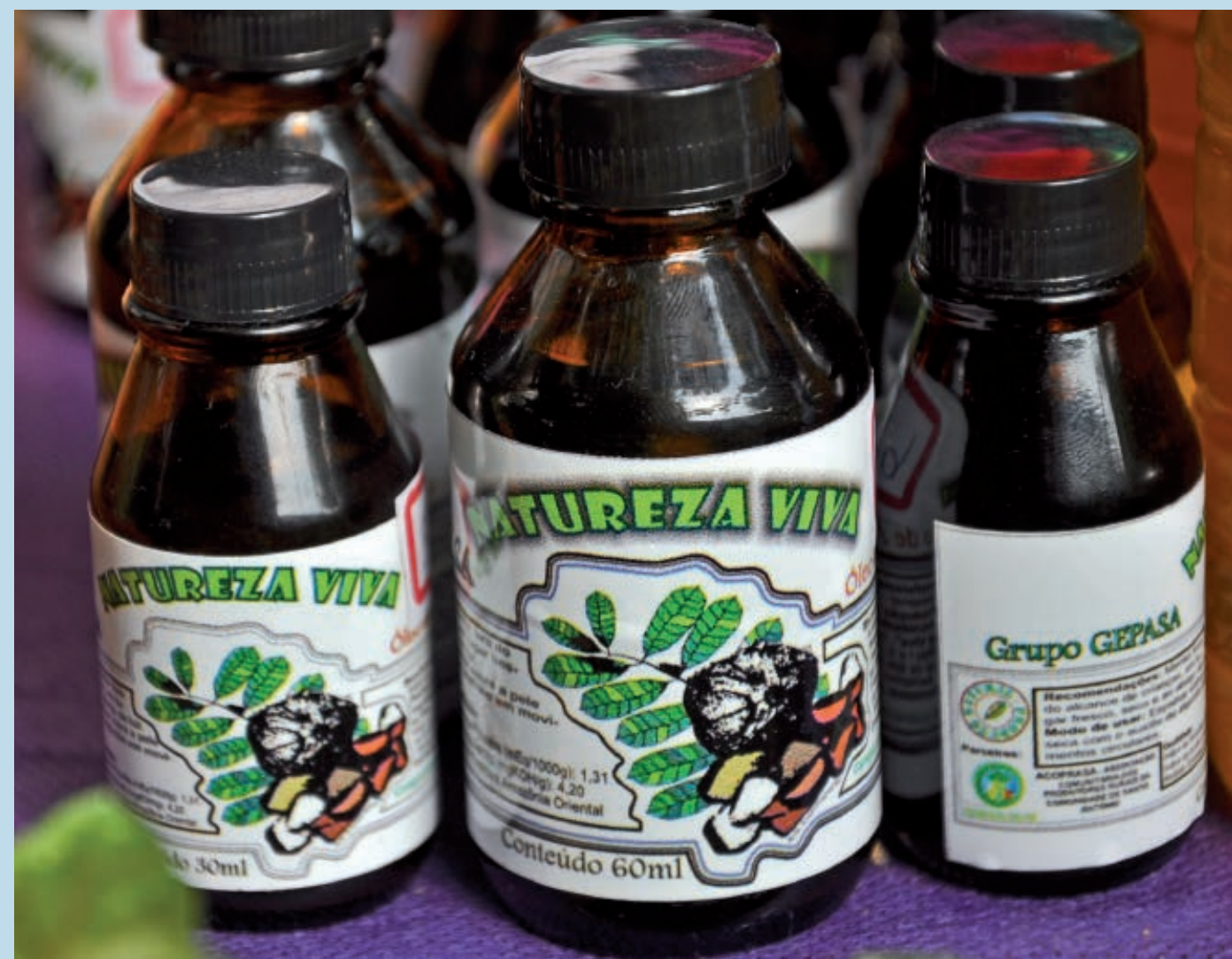

Bouteilles d'huile d'andiroba, Carapa guianensis, produites par l'association d'agriculteurs Natureza Viva de la communauté de Santo Antonio. Photo P. Sist. 


\section{RÉSUMÉ}

\section{LE POTENTIEL DE LA PRODUCTION FORESTIÈRE PAYSANNE EN AMAZONIE BRÉSILIENNE}

Dans l'État du Pará, 60 \% des forêts destinées à l'aménagement sont des forêts paysannes. Les concessions forestières d'entreprise ne pourront fournir au mieux que la moitié de la consommation actuelle. À quelques exceptions près, les communautés traditionnelles et les familles paysannes n'ont ni l'expérience technique ni la capacité économique pour conduire l'exploitation commerciale des bois : l'aménagement est réalisé par le biais d'accords avec une entreprise, selon différentes modalités. Dans ce contexte, les contrats entre communautés et entreprise sont de nature très variée et aboutissent au contrôle total par les entreprises privées de toutes les opérations, du plan d'aménagement à l'exploitation. Les politiques publiques et la législation forestière cherchent aujourd'hui à améliorer les capacités des communautés locales à défendre leurs intérêts dans le cadre de tels partenariats. Cette étude analyse le cadre financier de l'entreprise par rapport à l'organisation paysanne pour son exécution, et les éléments clés qui permettent un meilleur contrôle : les inventaires préexploitation, la diversification de la production de bois d'œuvre et de produits forestiers non ligneux, la commercialisation, le suivi et le contrôle des opérations d'exploitation. Il est proposé des pistes de politiques publiques qui doivent aider à la diffusion de l'aménagement paysan dans différentes contextes fonciers : unités de conservation et forêts de production, périmètres de colonisation agricole. L'article discute des modalités d'action et d'implantation d'un service public d'assistance technique et financière au profit des agriculteurs et des communautés. Le potentiel de l'intégration de la gestion forestière au sein des systèmes de production paysans est discuté dans le cadre du débat actuel de réforme du code forestier.

Mots-clés : gestion forestière communautaire, code forestier brésilien, Brésil, Amazonie.

\section{ABSTRACT}

\section{POTENTIAL OF PRODUCTION FOREST FARMER IN THE BRAZILIAN AMAZON}

In Brazil's Pará State, $60 \%$ of potentially managed forests are on community lands, while commercial timber concessions cannot cover more than half of current demand. With a few exceptions, traditional communities and smallholder families have neither the technical knowhow nor the economic capacity to conduct commercial logging operations: these are undertaken through agreements with a company under varying arrangements. Given this context, contracts between communities and companies are highly variable and always lead to the company exercising complete control over all operations, from management plans through to production. Public policies and land tenure regulations are now seeking to improve local community capacities for protecting their interests in partnerships of this kind. This study analyses how the financial business framework compares with smallholder organisation in the execution of these contracts, and addresses the key factors that could ensure better control: pre-logging inventories, diversification of production to include non-timber forest products as well as timber, marketing, monitoring and inspections of logging operations. Some possibilities of public policies are put forward to help disseminate the smallholder development under different land tenure regimes, such as conservation units in production forests or agricultural production boundaries. The article discusses ways in which a public service for technical and financial assistance to farmers and communities could act and become established. The potential for integrating forest management into small-scale farming systems is discussed in the light of the current debate on reform of the forest code.

Keywords: community forest management, Brazilian forest code, Brazil, Amazonia.

\section{RESUMEN}

\section{POTENCIAL DE LA PRODUCCIÓN FORESTAL CAMPESINA EN LA AMAZONIA BRASILEÑA}

En el Estado de Pará, el 60\% de los bosques destinados al manejo son bosques campesinos. Las concesiones forestales de empresas sólo podrán, como mucho, suministrar la mitad del consumo actual. Exceptuando algunos casos, las comunidades tradicionales y las familias campesinas no tienen ni la experiencia técnica ni la capacidad económica para dirigir el aprovechamiento comercial maderero: el manejo se realiza mediante acuerdos con una empresa según distintas modalidades. Dentro de este contexto, los contratos entre comunidades y empresas son de índole muy diversa y conducen al total control por las empresas privadas de todas las operaciones, del plan de manejo al aprovechamiento. Actualmente las políticas públicas y la legislación forestal intentan mejorar la capacidad de las comunidades locales para defender sus intereses en el marco de estos contratos. En este estudio, se analiza el marco financiero empresarial con relación a la organización campesina para su ejecución, así como los elementos clave que permiten un mejor control: inventarios pre-aprovechamiento, diversificación de la producción de madera de construcción y de productos forestales no madereros, comercialización, seguimiento y control de las operaciones de aprovechamiento. Se proponen ideas sobre políticas públicas encaminadas a ayudar a la difusión del manejo campesino en distintos marcos territoriales: unidades de conservación y bosques de producción, áreas de colonización agrícola. En el artículo se analizan los distintos modos de acción e implantación de un servicio público de asistencia técnica y financiera para ayudar a agricultores y comunidades. En el marco del actual debate de reforma del código forestal, se analiza el potencial de la integración de la ordenación forestal dentro de los sistemas de producción campesinos.

Palabras clave: manejo forestal comunitario, código forestal brasileño, Brasil, Amazonia. 


\section{Introduction}

L'aménagement des forêts naturelles représente aujourd'hui un des axes principaux du développement durable du milieu rural amazonien. Ne serait-ce que par l'extension des aires protégées et le pourcentage obligatoire des exploitations qui doit être maintenu en forêt, les politiques publiques tendent à positionner l'aménagement forestier comme une des seules alternatives de valorisation économique de vastes portions du territoire amazonien.

Pourtant, les limites de sa diffusion au Brésil sont connues : chaos foncier, concurrence du bois illégal, fragilité de l'administration et des organismes d'appui, fragilité technique et institutionnelle des acteurs de la filière. Si la loi de gestion des forêts publiques, adoptée en 2006, doit permettre de résoudre certaines de ces difficultés, notamment pour les entreprises, elle ne contribue que très peu à favoriser le développement d'une foresterie paysanne, pourtant nécessaire à la viabilisation économique de bien des zones rurales, et à l'approvisionnement de l'industrie du bois en Amazonie brésilienne. La diffusion de l'aménagement forestier paysan est donc le défi le plus actuel des politiques publiques dans cette région.

L'article analyse quelques expériences d'aménagement forestier paysan, relativement consolidées, de façon à identifier les principaux problèmes que pose leur diffusion. Quelques questions paraissent centrales dans cette problématique.

- Dans quelles conditions le développement d'une activité forestière au sein de systèmes de production traditionnellement axés sur l'agriculture représente-t-il un intérêt pour la paysannerie ? Ce point a été examiné dans le cadre de périmètres de colonisation individuelle de la région de Santarém (Pará), et le cas de l'exploitation forestière en faire-valoir direct, et celui de l'exploitation en sous-traitance à l'entreprise ont été analysés.

- Quelles sont les principales difficultés affrontées par les paysans et leurs organisations pour la mise en place de l'aménagement forestier? Quatre expériences de l'Ouest du Pará, qui exploitaient en 2008 leurs premières parcelles de forêt aménagée, ont été analysées de façon à rendre compte des problèmes techniques, financiers, institutionnels auxquels elles ont fait face.

- Comment des politiques publiques, dimensionnées de façon réaliste et cohérente avec les moyens dont elles disposent aujourd'hui, peuvent-elles réduire les principales limitations au développement de l'aménagement forestier paysan ? L'assistance technique et le financement ont été particulièrement discutés, et il a été proposé des modalités spécifiques d'intervention.

Le texte adopte ce cheminement en trois étapes, précisant pour chacune les données et les méthodes mobilisées.

\section{Les défis de la politique forestière dans le Pará}

\section{Le secteur forestier du Pará et ses problèmes}

L'État du Pará représente un quart de la surface de l'Amazonie brésilienne : $55 \%$ a aujourd'hui un statut d'aire protégée, Terre indigène ou Unité de conservation (ISA, IMAZON, 2011). Le défrichement accumulé représente aujourd'hui $20 \%$ de sa surface.

Le secteur forestier du Pará, dont le produit approche 1 milliard d'euros en 2009, consomme la moitié du bois de forêt naturelle de l'Amazonie brésilienne (SFB, IMAZON, 2010) ; il n'utilise que très marginalement le bois de forêts plantées. L'organisation non gouvernementale (Ong) Instituto do Homem e Meio Ambiente na Amazônia (Imazon) estime chaque année la part de l'exploitation illégale de bois, en rapprochant la localisation et la dimension des zones exploitées, détectées par satellite, des zones autorisées par le Secrétariat à l'environnement - Sema/PA. D'août 2009 à juillet 2010, 65 \% des surfaces exploitées l'ont été illégalement (78 941 ha sur un total de 120512 ha détectés). L'aménagement forestier légalisé, défini comme la seule alternative d'exploitation, se développe mais reste minoritaire (MONTEIRO et al., 2011).

L’importance toujours élevée de l'exploitation illégale dans le Pará peut être associée à une série de problèmes.

En premier lieu, pour les exploitants forestiers, la difficulté d'acquérir de nouveaux titres fonciers incontestables et les coûts de contrôle des terres en propriété découragent l'achat de forêts et finalement leur aménagement sur le long 
terme. Ce chaos foncier est difficile à réduire, de par les coûts nécessaires à l'intégration des cadastres existants dans les différents instituts publics à vocation foncière, à l'acquisition des cartes à l'échelle suffisante, à la collecte et l'enregistrement des prétendants au titre foncier, et finalement les coûts de la procédure judiciaire, souvent nécessaire pour trancher les superpositions de revendications foncières.

En deuxième lieu, l'informalité et la désorganisation du secteur forestier, le modèle prédateur qui domine la plupart des entreprises, rendent difficile leur adéquation aux pratiques légales d'une gestion durable. Ce secteur exige une gestion de long terme, de la ressource et des moyens économiques, un niveau technique plus conséquent. Sur les 1500 entreprises que compte le secteur forestier du Pará, l'Association des industries exportatrices de bois du Pará (Aimex) estime que moins de $10 \%$ sont capables de conduire un aménagement dans les conditions exigées par les pouvoirs publics (AIMEX, communication personnelle).

En troisième lieu, décentralisée en direction des États depuis 2006, la politique forestière n'a pas gagné suffisamment de consistance. Les compétences entre les administrations à vocation foncière ou environnementale, d'assistance technique et de crédit, ne sont pas stabilisées. Les ressources effectivement allouées aux contrôles de l'exploitation sur le terrain sont très faibles.

Enfin, généralement bénéficiaires d'une situation foncière mieux définie, les populations autochtones, ou les colons récents, pourraient en théorie adopter plus facilement l'aménagement forestier. Cependant, si l'on exclut certaines paysanneries de bords de fleuve, la paysannerie amazonienne ne dispose pas d'une tradition technique ni de la capacité économique d'exploitation commerciale du bois. Le modèle technique pris en compte par la législation, à savoir l'exploitation mécanisée pour la vente de grumes, les coûts d'obtention du permis d'exploiter (embauche d'un ingénieur forestier et suivi du dossier administratif), limitent l'aménagement des forêts communautaires à des expériences fortement subventionnées par projets, ou à des partenariats communautés-entreprises, généralement au bénéfice de ces dernières.

\section{Procédures inopérantes pour le contrôle} de l'exploitation

Concentration de l'exploitation sur de grandes surfaces cadastrées

\section{Loi de Gestion des Forêts Publiques (LGFP) : Concessions forestières}

Cadastre et mise en concession de forêts publiques

Difficulté d'accès à une propriété foncière sûre
Sélection rigoureuse des concessionnaires

Entreprises forestières peu structurées pour l'aménagement forestier
Figure 1.

Loi de gestion des forêts publiques comme stratégie de gestion du secteur forestier.

\section{Le développement nécessaire de l'aménagement en forêt paysanne}

La Loi fédérale de gestion des forêts publiques (Lgfp, $\left.n^{\circ} 11.284,2006\right)$ représente la principale stratégie gouvernementale pour le secteur forestier amazonien au Brésil. Elle définit les attributions respectives des administrations fédérales et des États, crée le Service forestier brésilien $(\mathrm{Sfb})$, le registre national des forêts publiques, et définit les modalités de leur mise en concession.

La mise en concession de forêts publiques doit donc faire progresser l'aménagement forestier durable légalisé parmi les entreprises à travers les mécanismes suivants :

- la diffusion des concessions doit résoudre pour les entreprises la question de l'accès sécurisé aux ressources sur le long terme ;

- doivent être sélectionnées des entreprises capables de réaliser un aménagement de qualité, et le volume des concessions prévu doit aider à remodeler le secteur comme un tout ; - la concentration des concessions au sein d'unités de grande dimension, et les ressources prévues dans la loi pour la financer, doivent faciliter la routine de contrôle public de l'exploitation (figure 1).

Depuis 2007, cinq procédures de mise en concession ont été finalisées dans deux forêts nationales (Flona Jamari et Saracá-Taquera), et trois autres ont été soumises à consultation publique (Flona Amana). La surface totale des Flona considérées est de 1206003 ha, et la surface totale des dix concessions est de 448757 ha (SFB, 2011, 2012) : ces concessions occupent donc en moyenne un tiers de la surface des Flona et 44876 ha chacune. Or, un rapide calcul, basé sur l'estimation de la consommation annuelle de bois de forêts naturelles dans le Pará (6,6 millions de m³ en 2009 ; PEREIRA et al., 2010), indique qu'il faudrait une surface de forêts aménagées de 9,9 millions d'hectares, si l'on admet les paramètres communs d'un cycle de 30 ans et d'une intensité de coupe de $20 \mathrm{~m}^{3} /$ ha dans les parcelles exploitées.

Les politiques de destination des terres en Amazonie brésilienne établissent un stock de forêts plus facilement disponibles pour l'aménagement, soit des zones où la question foncière n'est pas un obstacle. Il s'agit des :

- Forêts nationales (Flona), et des États (Flota) ;

- Réserves de développement durable (Rds) des États, et des Réserves extractivistes (Resex), fédérales ;

- Périmètres de réforme agraire, individuels (Pas) et collectifs (Pds) ;

- Forêts publiques du Pará enregistrées (« glèbes », glebas arrecadadas) mais non destinées. Nous ne comptabilisons que celles sous la juridiction de l'État du Pará (IDEFLOR, 2009).

Alors que l'ensemble de ces forêts peuvent être aménagées par les communautés paysannes, seules les forêts destinées à la production (Flona, Flota) et les " glèbes" peuvent être mises en concession pour des entreprises, dans le cadre de plans de gestion et de zonages élaborés pour l'ensemble de leur surface. L'aménagement forestier des périmètres de réforme agraire est possible au sein des réserves forestières obligatoires - áreas de reserva légal (Arl), fixées à 50-80 \% de la propriété selon les régions de l'État. Les Terres indigènes ont été exclues du total, car la législation décourage fortement leur aménagement. 
Tableau I.

Potentiel de production des forêts destinées ou potentiellement destinées à l'aménagement (Pará).

\begin{tabular}{|c|c|c|c|c|c|c|c|c|c|}
\hline Pará & \multicolumn{2}{|c|}{$\begin{array}{l}\text { Surface totale: } \\
124800000 \text { ha }\end{array}$} & \multicolumn{5}{|c|}{ Surface estimée pour l'aménagement } & \multicolumn{2}{|c|}{$\begin{array}{c}\text { Volume potentie } \\
\text { de bois }\end{array}$} \\
\hline \multirow[t]{2}{*}{ Statut des terres } & \multirow[t]{2}{*}{$\begin{array}{l}\text { Surface (2) } \\
\text { (ha) }\end{array}$} & \multirow[t]{2}{*}{$\%$} & \multicolumn{2}{|c|}{$\begin{array}{l}\text { Aménagement } \\
\text { d'entreprise }\end{array}$} & \multicolumn{2}{|c|}{$\begin{array}{l}\text { Aménagement } \\
\text { communautaire }\end{array}$} & \multirow{2}{*}{$\begin{array}{c}\text { Total } \\
\text { ha }\end{array}$} & & \\
\hline & & & $\%$ de (2) & & $\%$ de (2) & ha & & $m^{3}$ & $\%$ \\
\hline Flota & 7862321 & 6,3 & 30 & 2358696 & 20 & 1572464 & 3931160 & 2620774 & 33 \\
\hline Flona & 6292509 & 5,0 & 30 & 1887753 & 20 & 1258502 & 3146255 & 2097503 & 26 \\
\hline Rds & 129912 & 0,1 & & & 30 & 38974 & 38974 & 25982 & 0 \\
\hline Resex & 4097950 & 3,3 & & & 30 & 1229385 & 1229385 & 819590 & 10 \\
\hline Pds & 2195450 & 1,8 & & & 30 & 658635 & 658635 & 439090 & 6 \\
\hline PA & 7745396 & 6,2 & & & 30 & 2323619 & 2323619 & 1549079 & 20 \\
\hline Glèbes (Iterpa) & 1172384 & 0,9 & 50 & 586192 & & & 586192 & 390795 & 5 \\
\hline Total (ha) & 29495922 & 23,6 & & 4832641 & & 7081578 & 11914219 & 7942813 & 100 \\
\hline \multicolumn{3}{|c|}{ Volume potentiel de bois $\left(\mathrm{m}^{3}\right)$} & & 3221761 & & 4721052 & 7942813 & & \\
\hline \multicolumn{3}{|l|}{ Pourcentage } & & $41 \%$ & & $59 \%$ & & & \\
\hline
\end{tabular}

Hypothèse : intensité de coupe 20 m³/ha, cycle 30 ans, soit une production de 0,67 m³/ha/an (IDEFLOR, 2009; SFB, 2009; IMAZON, communication personnelle, 2009 ; calculs des auteurs).

Le tableau I montre notre calcul de potentiel de production pour ces forêts, et particulièrement l'importance relative de l'aménagement communautaire dans le total.

Les hypothèses sur les parts relatives des surfaces sous aménagement sont optimistes (50\% des Flona, Flota, Glebas, $30 \%$ des Rds, Resex et Pas, Pds) en regard de la situation actuelle, car elles sont cohérentes avec la stratégie publique mise en œuvre à partir de 2006. Celles relatives à l'intensité de coupe $\left(20 \mathrm{~m}^{3} / \mathrm{ha}\right)$ et à la durée du cycle (30 ans) sont des moyennes observées actuellement (SIST, NASCIMENTO-FERREIRA, 2007). Les $50 \%$ des Flota et Flona sous aménagement ont été réparties en $30 \%$ pour les entreprises (ce qui existe actuellement) et $20 \%$ pour les communautés.

Ainsi, la surface potentielle sous aménagement dans ces zones est d'environ 12 millions d'hectares (tableau I), et permet l'approvisionnement du secteur forestier un peu audelà de son niveau actuel (environ 8 millions de mètres cubes pour un besoin de 6,6 millions). Mais la part de l'aménagement communautaire dans ces chiffres est de $59 \%$, contre $41 \%$ pour l'aménagement d'entreprise : l'aménagement communautaire est nécessaire à l'approvisionnement du secteur forestier dans le Pará.

\section{Potentiels et obstacles à l'aménagement communautaire pour la production de bois}

\section{Le fragile développement de l'aménagement communautaire}

En 2006, il y avait 1566 plans d'aménagement communautaires soumis à l'administration, principalement des plans de petite dimension à gestion individuelle $(n=1389)$, et en moindre proportion des plans collectifs $(n=176)$, en majorité situés autour ou dans les unités de conservation. La surface correspondante dans le Pará était alors de 212948 ha (44 plans à gestion collective, 566 à gestion individuelle) (SFB, 2007). Trois ans plus tard (2009-10), les plans à gestion collective sont moins nombreux dans les États de l'Acre, du Pará, de l'Amazonas, du Rondônia (passant de 142 à 123). Les chiffres pour les plans individuels sont indisponibles (PINTO et al., 2011). Mais ces chiffres sont peu représentatifs quant à la mise en œuvre effective de l'exploitation. Un séminaire récent montrait que les autorisations bénéficiaient à moins de $20 \%$ des plans dans l'Amazonas (CNS, IDESAM, 2010). Il est notoire aussi que la détention des plans par les communautés ou les paysans individuels ne signifie pas forcément qu'ils gèrent directement l'aménagement (CRUZ et al., 2011). Ces indications montrent le caractère fragile et réduit de la contribution des communautés paysannes au secteur forestier pour le bois. 
Cela est dû à plusieurs raisons.

- Les régions de bord de fleuve (ribeiras) constituent les seules zones où la paysannerie a une expérience historique dans l'exploitation des bois. Elles souffrent de la rétraction du marché des bois légers, concurrencés au niveau national par les plantations, et de difficultés spécifiques en matière de légalisation foncière (attributions confuses entre administrations et conflits latents avec les élites traditionnelles). - Pour ce qui est des autres zones (terra firme), le modèle technique dominant et les filières correspondantes, qui privilégient l'extraction mécanisée de grumes d'un nombre réduit d'espèces destinées au marché national ou à l'exportation, sont particulièrement inadaptés du point de vue paysan : le volume de capital nécessaire, la capacité technique et de gestion pour ce type d'activité, éventuellement la capacité de contrôle des contrats avec les entreprises, ne lui sont accessibles que par le biais de projets subventionnés.

- Les coûts d'obtention des autorisations administratives sont élevés et exigent des investissements préalables : licence environnementale des propriétés, légalisation des organisations de base, embauche d'un ingénieur forestier, réalisation des inventaires, suivi du dossier administratif (dans certains cas, plusieurs années).

Au final, les expériences d'aménagement paysan des forêts qui fonctionnent ont lieu sur des terres légalisées ou sur des zones pilotes bénéficiant de régimes fonciers aménagés et ont bénéficié de subventions importantes en financement et appui technique, soit par le biais de projets spécifiques publics ou de type Ong, soit par la mise en place d'un partenariat avec des entreprises (CRUZ et al., 2011 ; PINTO et al., 2011).

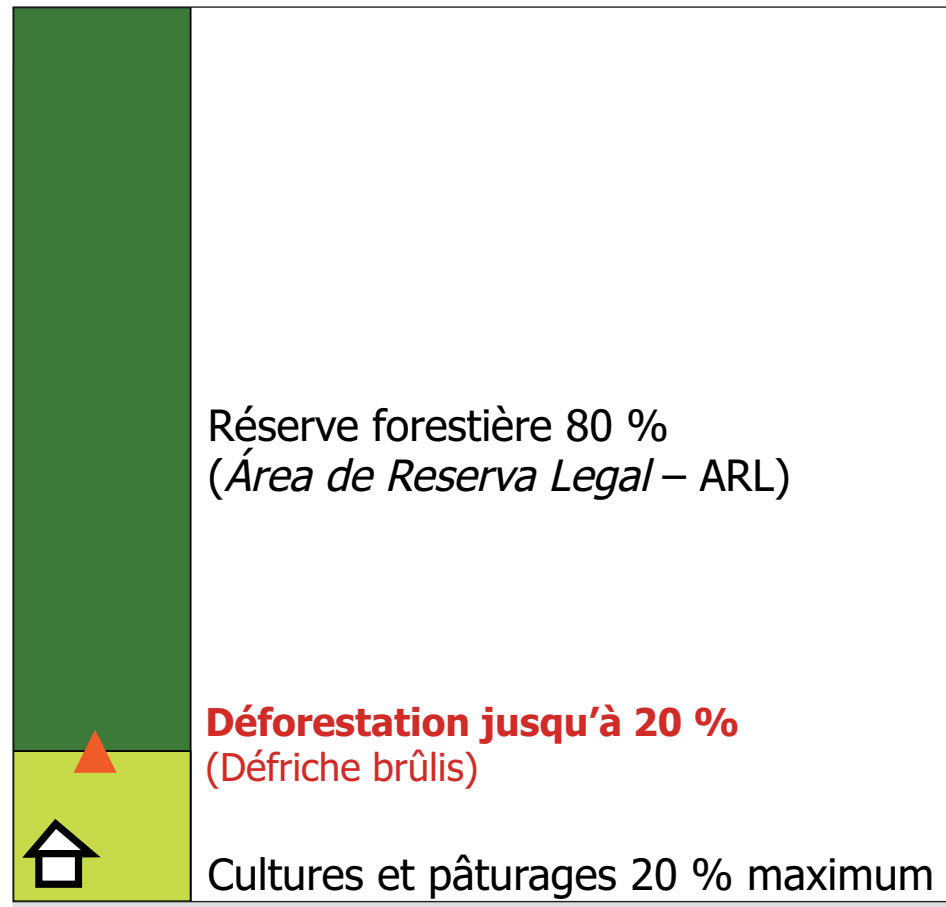

Figure 2.

La réserve forestière obligatoire au sein des exploitations agricoles amazoniennes.

\section{La production paysanne de bois de forêt naturelle dans les périmètres de colonisation}

\section{Des systèmes de production agriculture-forêt}

Pour analyser l'intérêt de l'agriculteur à exploiter le bois au sein de sa propriété dans les conditions actuelles, le périmètre de réforme agraire PA Mojú est pris en exemple, dans la région de Santarém, où l'aménagement est effectué par le biais d'accords entre les associations paysannes, détentrices des plans d'aménagement, et une entreprise, Maflops Ltda. Le fait que cette expérience n'ait pas été subventionnée a permis de mieux identifier les coûts et le cadre financier de l'accord (CRUZ et al., 2011).

La construction de la route BR 163 dès 1973-1976, et la colonisation publique menée sur ses marges par l'Institut national de la colonisation et la réforme agraire (Incra), conditionnent l'histoire postérieure de l'occupation paysanne du PA Mojú. L'Incra installe les colons sur des lots de 100 ha $(400 \times 2500 \mathrm{~m})$, d'abord sur une frange de $10 \mathrm{~km}$ à l'Est de la route. Selon le code forestier de l'époque, chaque famille devait maintenir $50 \%$ de son lot en réserve forestière. En 2000, l'Incra crée le PA Mojú, au-delà de cette frange de $10 \mathrm{~km}$; en 2009, ce périmètre de 146000 ha compte 400 familles bénéficiaires, et montre un taux relativement faible de défrichement ( $12 \%$ de la surface).

Le gouvernement fédéral a fait passer la surface obligatoire en forêt des exploitations à 80 \% en 2001 : les colons doivent donc mettre en place des systèmes de production mixtes forêt-agriculture sur respectivement $80 \%$ et $20 \%$ de leurs surfaces (figure 2).

Au début des années 2000, l'Incra (et les autres institutions publiques responsables) a accepté la signature d'accords entre les associations de colons et une entreprise locale d'exploitation forestière, Maflops Ldta : Maflops devait construire les routes, préparer les documents et exécuter le plan d'aménagement détenu par les associations signataires des accords, et payer à chaque colon, dont le lot serait exploité, un montant proportionnel au volume de bois extrait. En 2006, Maflops avait établi des contrats avec 13 associations, dont neuf avaient obtenu les autorisations d'exploitation, totalisant 45000 ha. Chaque plan d'aménagement d'association regroupe de 40 à 60 familles. L'exploitation, sur la base d'un cycle de 30 ans, est réalisée en deux à six coupes, chacune regroupant les surfaces en forêt de 10 à 20 lots individuels.

Pour l'année 2009, les résultats économiques des activités agricoles de 79 exploitants de deux associations du PA Mojú, qui ont signé un contrat avec Maflops (les associations Acoprasa et Aprocosma), ont été analysés. Une typologie des systèmes de production et leur analyse économique ont été élaborées, selon les principes présentés dans DUFUMIER (1996). Ainsi les revenus potentiels sur les $20 \%$ de la surface qui peuvent être légalement défrichés (BARTHELON, 2009) ont été évalués. Ensuite, les résultats de l'exploitation de bois en 2008 et d'huile d'andiroba (Carapa guianensis Aubl.) en 2010 ont été analysés sur une partie des lots d'une de ces deux associations, l'Acoprasa. De cette façon, le revenu potentiel des $80 \%$ de la surface de réserve forestière obligatoire a pu être évalué et comparé avec les revenus agricoles (CRUZ et al., 2011). 
Les résultats économiques des activités agricoles

Le tableau II montre les résultats économiques des six principaux systèmes de production identifiés dans les enquêtes. Chacun est modélisé pour la surface maximale par unité de travail agricole (Uta), les indicateurs étant : le revenu agricole (RA) maximal par Uta (RA/Uta), le revenu par hectare du système $(R A / h a)$, et la fréquence du système considéré parmi les enquêtés. Les revenus sont donnés en monnaie courante. Nous utilisons la fréquence pour calculer la moyenne pondérée de RA/Uta parmi les enquêtés : ce chiffre représente le revenu moyen par travailleur accessible dans les conditions de production du PA Mojú.

\section{Les résultats économiques de l'exploitation forestière et du système agriculture-forêt}

Les activités forestières analysées pour le partenariat Acoprasa/Maflops recouvrent l'extraction et la vente de grumes en 2008 (606 ha de forêt au total sur 11 lots individuels), la collecte et la production d'huile d'andiroba en 2010, que nous avons pu accompagner sur le terrain ; ensuite, ont été estimés les résultats d'une possible exploitation des résidus de bois sur la parcelle d'où ont été retirées les grumes. Le tableau IIla présente les résultats économiques du point de vue des colons, pour une superficie moyenne de forêt aménagée de 60 ha, dans le cadre du contrat avec Maflops pour les grumes et les résidus, et de la production d'huile d'andiroba en exploitation directe. Ensuite les résultats de l'exploitation directe du bois et de l'andiroba ont été estimés pour les colons, en faisant l'hypothèse qu'ils maintiendraient les indices technico-économiques de Maflops (tableau IIIb). II a été vérifié que le volume de travail nécessaire pour l'exploitation directe est facilement mobilisable : la production de grumes et de résidus requiert pour le cycle environ 400 jours de travail, au moment de l'exploitation des 60 ha, soit 15 jours par an ; la production annuelle d'andiroba consomme 35 jours, le total annuel se situe donc à 50 jours (0,2 Uta).

Ces résultats et leur comparaison avec ceux des activités agricoles montrent ce qui suit.

- La production de bois est la production forestière principale. La production d'huile ne fournit qu'un revenu marginal. L'exploitation directe du bois par les colons permet de quadrupler le revenu obtenu dans le cadre du contrat avec l'entreprise, ce qui rend l'alternative intéressante, vu le faible volume de main-d'œuvre nécessaire (15 jours par an).

- Même conduite dans de bonnes conditions (mêmes résultats technico-économiques que l'entreprise spécialisée), l'exploitation forestière directe est moins rémunératrice que l'agriculture (revenu de 142,4 R\$/ha contre $371 \mathrm{R} \$ /$ ha). Elle dépasse à peine le revenu du système 1 (cultures annuelles, $114 \mathrm{R} \$ / \mathrm{ha}$ ), le plus décapitalisé, et du système 6 (élevage spécialisé, 135 R\$/ha), le plus extensif. Ainsi, une fragilisation du contrôle public sur le maintien des surfaces obligatoires en forêt doit conduire à leur défrichement à des fins agricoles.

- Mais dans le cadre du maintien de ce contrôle, les colons ont intérêt à mettre en œuvre l'aménagement forestier. Le revenu agricole moyen de 20 ha est de 7 420,00 R \$ (20x $371 \mathrm{R} \$$ ), soit 1,2 salaire minimum. L'exploitation forestière de 60 ha par contrat d'entreprise fournit un revenu additionnel de 2 496,00 R\$, et $8544,00 \mathrm{R} \$$ en exploitation directe : dans ce dernier cas, le revenu est plus que doublé.

\section{Tableau II. Résultats économiques de l'agriculture dans le PA Mojú (2009).}

Principaux systèmes

de production

Cultures annuelles

d'autoconsommation

$(2)=(1)+$ cultures de manioc commercial

(3) $=(2)+$ poivre, cacao, safran

(4) = Spécialisés poivre*

(5) = (3) + élevage bovin (extensif)

(5) = (3) + élevage bovin

(intensif)

(6) = Éleveurs spécialisés
Description du modèle par Uta

8 ha dont 1 ha de cultures annuelles et 7 ha de friches en rotation. Riz, maïs et volailles, avec des rendements faibles.

8 ha dont 1 ha de cultures annuelles et 7 ha de friches en rotation. Riz, maïs, manioc et volailles, avec de bons rendements.

10 ha dont 1 ha de cultures annuelles, 0,25 ha de poivre et 8,75 ha de friches en rotation. Riz, maïs, manioc et volailles, avec de bons rendements.

8 ha, dont 4 ha de poivre (10 000 pieds) et 4 ha de friches en rotation.

28 ha dont 0,5 ha de cultures annuelles, 1 ha de poivre, 20 ha de pâturage, 6 ha de friches en rotation.

24 ha dont 0,5 ha de cultures annuelles, 1 ha de poivre, 16 ha de pâturage, 6 ha de friches en rotation.

40 ha de pâturage.

RA/ha moyenne sur zone

\begin{tabular}{c|c|c}
\hline $\begin{array}{c}\text { RA/Uta } \\
\text { maximale }\end{array}$ & $\begin{array}{c}\text { RA/ha du } \\
\text { système }\end{array}$ & $\begin{array}{r}\text { Fréquence } \\
\text { enquêtés }\end{array}$ \\
\hline 911 & 114 & $10 \%$ \\
\hline 3220 & 403 & $18 \%$ \\
\hline 4669 & 467 & $39 \%$ \\
\hline 20352 & 2544 & $1 \%$ \\
\hline 7149 & 255 & $11 \%$ \\
\hline 8441 & 352 & $11 \%$ \\
\hline 5400 & 135 & $10 \%$ \\
\hline
\end{tabular}

* Le travail total correspond à 650 jours, dont 260 familiaux (1 Uta), et 390 salariés (payés 20 R\$ par jour) comptabilisés dans les coûts. Les cinq autres modèles n'emploient que du travail familial. Par exemple, le système de production 3 (cultures annuelles, manioc, cultures pérennes), qui concerne $39 \%$ des enquêtés, occupe un travailleur à chaque 10 ha, le rémunérant à 4669 R\$ par an, soit trois quarts d'un salaire minimum. En 2009, l'euro s'échangeait autour de 2,8 Reals. 
Tableaux IIla et IIIb.

Résultats économiques de l'exploitation forestière dans le PA Mojú (Acoprasa, Maflops, 2008) dans le cas d'un partenariat avec l'entreprise Maflops (IIIa) et sans partenariat (IIIb).

\section{Tableau IIla.}

\begin{tabular}{|c|c|c|c|}
\hline Activités forestières & Description des activités & $\begin{array}{l}\text { Revenu/lot, } \\
\text { cycle (R\$) }\end{array}$ & $\begin{array}{l}\text { Revenu/ha } \\
\text { an (R\$) }\end{array}$ \\
\hline $\begin{array}{l}\text { (1) = Production de grumes (contrat } \\
\text { association/entreprise) }\end{array}$ & $\begin{array}{l}\text { L'entreprise finance, exécute l'exploitation forestière pour le compte des } \\
\text { colons et leur paie } 25 \% \text { du revenu correspondant. Le cycle de coupe est } \\
\text { de } 30 \text { ans, } 60 \text { ha de forêt aménagée par colon, coupée en une fois. }\end{array}$ & 40320 & 22,4 \\
\hline $\begin{array}{l}\text { (3) = Production d'huile d'andiroba } \\
\text { (exploitation directe) }\end{array}$ & $\begin{array}{l}\text { Les colons collectent, produisent et commercialisent l’huile, et se répar- } \\
\text { tissent le revenu correspondant. La surface de collecte est de } 60 \text { ha par } \\
\text { famille, et la production est annuelle. }\end{array}$ & 14400 & 8 \\
\hline & Revenu total/ha/an & & $41,6 R \$$ \\
\hline
\end{tabular}

Tableau IIIb.

Activités forestières

(1) = Production de grumes (exploitation directe)

(2) = Production de résidus (exploitation directe) - estimée

(3) = Production d'huile d'andiroba (exploitation directe)
Description des activités

Les colons financent, exécutent l'exploitation forestière et se répartissent le revenu correspondant. Le cycle de coupe est de 30 ans, 60 ha de forêt aménagée par colon, coupée en une fois.

Idem ci-dessus.

Les colons collectent, produisent et commercialisent l'huile, et se répartissent le revenu correspondant. La surface de collecte est de 60 ha par famille, et la production est annuelle.

Revenu total/ha/an

\begin{tabular}{c|c}
$\begin{array}{c}\text { Revenu/lot, } \\
\text { cycle (R\$) }\end{array}$ & $\begin{array}{r}\text { Revenu/ha } \\
\text { an (R\$) }\end{array}$ \\
\hline 161280 & 89,6 \\
\hline 80640 & 44,8 \\
\hline 14400 & 8 \\
& $142,4 \mathrm{R} \$$
\end{tabular}

\section{La problématique de l'exploitation directe des forêts par les paysans}

Une fois vérifié le potentiel de l'aménagement forestier par les colons, il s'agit d'analyser par quels biais ils peuvent acquérir le métier d'exploitant forestier, et ce qui doit favoriser la durabilité d'un aménagement forestier paysan.

Pour ce faire, l'analyse du partenariat AcoprasaMaflops et celle de trois autres expériences d'aménagement communautaire de l'Ouest du Pará sont reprises, en relation avec les entreprises à des degrés différents, mais toutes visant la commercialisation de grumes. Le plan d'aménagement y est en phase d'exécution, l'organisation communautaire participe aux opérations et contrôle de plus ou moins près la commercialisation. Ces trois autres expériences sont celles de la Cooperativa Mista Flona Tapajós Verde (Coomflona), située dans la Flona Tapajós (Belterra-PA), de la Cooperativa Agroextrativista Novos Rumos (Canor), à Uruará-PA, de l'association du Projeto de Desenvolvimento
Sustentável - Pds Virola-Jatobá, à Anapú-PA (CRUZ et al., 2011 ; DRIGO et al., 2009).

L'aménagement est exécuté directement par la Coomflona et la Canor, et par le biais d'entreprises à l'Acoprasa et au Pds Virola-Jatobá. La propriété foncière et des ressources forestières est individuelle à l'Acoprasa et à la Canor (lots individuels de colonisation), et collective à la Coomflona (forêt publique fédérale) et au Pds Virola-Jatobá (périmètre collectif de colonisation ; tableau IV).

L'analyse de ces exemples permet d'identifier certaines des conditions qui doivent favoriser la durabilité et la gouvernance de l'aménagement forestier paysan.

- L'implication d'une assistance technique sur le moyen/long terme, notamment pour l'appui aux organisations de base. Les trois expériences avec une vraie participation paysanne dans l'exploitation ou la gestion (Coomflona, Canor, Pds Virola-Jatobá) ont eu un véritable appui technique et de formation pendant plusieurs années. 
Tableau IV.

Caractérisation de quatre expériences d’aménagement paysan dans l’Ouest du Pará (CRUZ et al., 2011).

\begin{tabular}{|c|c|c|c|c|}
\hline Expériences & $\begin{array}{l}\text { Description du plan } \\
\text { d'aménagement }\end{array}$ & Résultats économiques 2008 & Points favorables & Difficultés \\
\hline Coomflona & $\begin{array}{l}\text { Forêt publique } \\
\text { Surface plan = } 32000 \text { ha } \\
\text { Exploitation en parcelles } \\
121 \text { associés (2007) } \\
\text { Faire-valoir direct }\end{array}$ & $\begin{array}{l}\text { Exploitation }(2008): \\
\text { Surface }=500 \mathrm{ha} \\
\text { Intensité }=11,4 \mathrm{~m}^{3} / \mathrm{ha} \\
\text { Nombre espèces }=18 \\
\text { Prix moyen }=190 \mathrm{R} \$ / \mathrm{m}^{3} \\
\text { Revenu }=33 \mathrm{R} \$ / \mathrm{ha} / \mathrm{an}\end{array}$ & $\begin{array}{l}\text { Appui financier et } 10 \text { ans de } \\
\text { formation technique } \\
\text { Commercialisation supervisée } \\
\text { par Ibama } \\
\text { Contrôle de l'exploitation } \\
\text { illégale par le gouvernement }\end{array}$ & $\begin{array}{l}\text { Gestion des coûts : gestion } \\
\text { des droits des habitants de la } \\
\text { Flona, coût (élevé) des } \\
\text { équipements loués } \\
\text { Espèces hors exportation } \\
\text { difficiles à vendre } \\
40 \% \text { du bois inventorié non } \\
\text { exploitable }\end{array}$ \\
\hline Canor & $\begin{array}{l}\text { Colonisation individuelle } \\
\text { Surface plan = } 364 \text { ha } \\
\text { Exploitation en parcelles } \\
6 \text { familles dans le plan } \\
\text { Faire-valoir direct }\end{array}$ & $\begin{array}{l}\text { Exploitation }(2008): \\
\text { Surface }=74 \text { ha } \\
\text { Intensité }=14 \mathrm{~m}^{3} / \mathrm{ha} \\
\text { Nombre espèces }=21 \\
\text { Prix moyen }=115 \mathrm{R} \$ / \mathrm{m}^{3} \\
\text { Revenu }=11 \mathrm{R} \$ / \mathrm{ha} / \mathrm{an}\end{array}$ & $\begin{array}{l}\text { Connaissance antérieure de } \\
\text { l'exploitation forestière par } \\
\text { les familles qui ont assuré } \\
\text { directement l'exploitation }\end{array}$ & $\begin{array}{l}\text { Peu d'acheteurs potentiels } \\
\text { sur la zone, qui imposent } \\
\text { leurs conditions } \\
\text { Lenteur de la procédure de } \\
\text { licence (plusieurs années) }\end{array}$ \\
\hline $\begin{array}{l}\text { Pds Virola- } \\
\text { Jatobá }\end{array}$ & $\begin{array}{l}\text { Colonisation en terre collective } \\
\text { Surface plan = } 23467 \text { ha } \\
\text { Exploitation en parcelles } \\
80 \text { familles dans l'association } \\
\text { Exploitation à l'entreprise }\end{array}$ & $\begin{array}{l}\text { Exploitation }(2008): \\
\text { Surface }=500 \text { ha } \\
\text { Intensité }=8 \mathrm{~m}^{3} / \mathrm{ha} \\
\text { Nombre espèces }=28 \\
\text { Prix moyen }=\mathrm{s} . \mathrm{d} . \\
\text { Revenu }=13 \mathrm{R} \$ / \mathrm{ha} / \mathrm{an}\end{array}$ & $\begin{array}{l}\text { Appui financier et partenaires } \\
\text { techniques actifs, qui } \\
\text { accompagnent contrats et } \\
\text { commercialisation } \\
\text { Les colons ont réalisé } \\
\text { l'inventaire }\end{array}$ & $\begin{array}{l}\text { Lenteur de l'Incra } \\
\text { Fragilité de l'association par } \\
\text { rapport à l'entreprise } \\
\text { Difficulté du contrôle } \\
\text { de l'exploitation illégale } \\
50 \% \text { du bois inventorié n'est } \\
\text { pas exploitable }\end{array}$ \\
\hline Acoprasa & $\begin{array}{l}\text { Colonisation individuelle } \\
\text { Surface plan = } 2510 \text { ha } \\
\text { Exploitation en une fois } \\
42 \text { familles dans le plan } \\
\text { Exploitation à l'entreprise }\end{array}$ & $\begin{array}{l}\text { Exploitation (2008) } \\
\text { Surface }=606 \text { ha } \\
\text { Intensité }=24,4 \mathrm{~m}^{3} / \mathrm{ha} \\
\text { Nombre espèces }=41 \\
\text { Prix moyen }=\mathrm{s} . \mathrm{d} . \\
\text { Revenu }=22 \mathrm{R} \$ / \text { ha/an }\end{array}$ & $\begin{array}{l}\text { Bons résultats économiques } \\
\text { (dus à l'intensité de coupe). } \\
\text { Le contrôle de la commerciali- } \\
\text { sation par l'entreprise et un } \\
\text { meilleur emploi du bois } \\
\text { inventorié autorisent une plus } \\
\text { grande intensité de coupe }\end{array}$ & $\begin{array}{l}\text { Difficultés dans le contrôle de } \\
\text { l'exploitation illégale } \\
\text { Association peu consolidée } \\
\text { (pas de ressources et peu de } \\
\text { soutien des colons) et donc } \\
\text { peu de gouvernance }\end{array}$ \\
\hline
\end{tabular}

L’appui à l'organisation paysanne détentrice juridique du plan d'aménagement, et sa gestionnaire technique et financière, est fondamental, sous peine de voir sa fragilisation s'étendre à la gouvernance de l'aménagement.

- Les expériences consolidées ont reçu un appui financier significatif direct, par la mise à disposition d'ingénieurs forestiers avant l'exploitation, pour l'élaboration et l'obtention de la licence d'exploitation, par l'achat de quelques équipements. La Coomflona s'est aussi vu financer l'exploitation de sa première parcelle.

- Dans tous les cas l'importance du contrôle réel de la commercialisation est mesurée comme composante clé du revenu et de la gouvernance de l'exploitation forestière. Il commence avant l'exploitation, car le choix des espèces à couper doit tenir compte du marché et des prix, et la vente elle-même peut être négociée sur la base du volume de coupe autorisé, et ainsi donner lieu à un paiement anticipé qui aide au financement de l'exploitation. La gouvernance de l'aménagement est très liée au contrôle administratif du volume de bois extrait, de sa vente à différents acheteurs et de son transport. On voit la différence des prix obtenus la même année par la Coomflona et la Canor : la Coomflona a bénéficié de la super- vision de la commercialisation par l'Ibama, et la Canor ne s'est préoccupée de la vente de son bois qu'après exploitation. Pour l'Acoprasa, l'entreprise Maflops gère l'essentiel des fonctions de commercialisation et parvient à commercialiser un nombre plus important d'espèces.

- Le rôle des pouvoirs publics est fondamental pour la gouvernance de l'aménagement paysan, dans ce contexte où les acteurs sont si inégaux : procédures de licence simplifiées et moins coûteuses à mettre en place, contrôle de l'exploitation illégale, supervision et suivi des contrats (ou simplement de la commercialisation), appui effectif aux colons (infrastructures, appui technique, financement, etc.).

- La relation avec les entreprises a des effets contrastés. L'Acoprasa montre qu'elle permet de viabiliser l'aménagement et l'obtention d'un revenu significatif. Mais sans intervention externe, cette relation enferme les paysans dans la dépendance et un modèle d'exploitation peu durable et à court terme (grumes d'espèces pour les marchés nationaux ou internationaux). Elle défavorise la mise en place de modèles plus accessibles à une gestion paysanne autonome, basés sur la transformation locale et l'approvisionnement des marchés locaux. 


\section{Les politiques publiques pour appuyer le développement de l'aménagement forestier paysan}
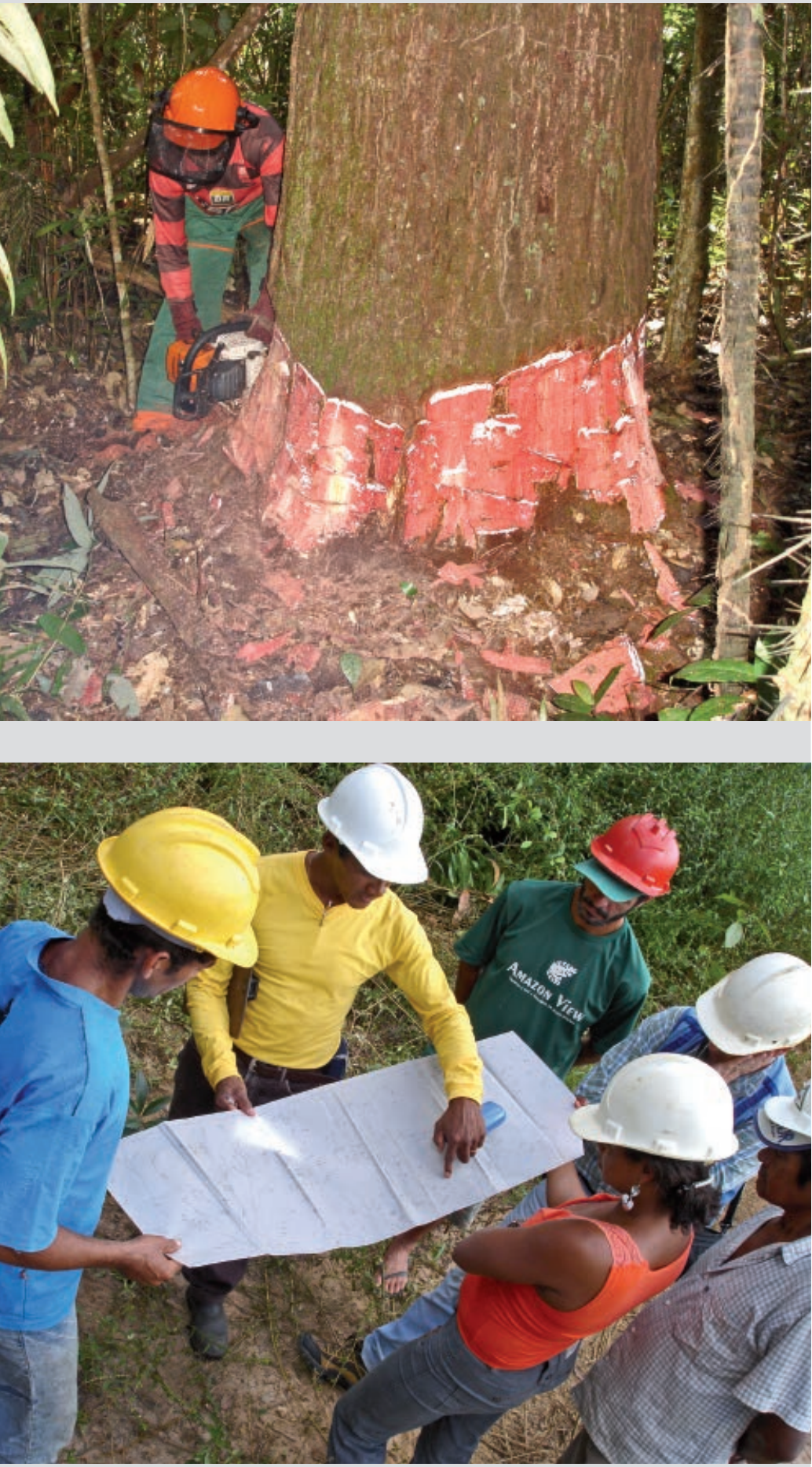

Abattage d'un arbre de la famille des sapotacées selon les techniques recommandées par l'exploitation à faible impact (RIL-reduce impact logging; niveau de la coupe à la base du tronc afin de valoriser au maximum le volume de bois récolté) suite à la sélection cartographique issue de l'inventaire d'exploitation. Photos P. Sist.
Idéalement, un contexte plus favorable à l'aménagement forestier paysan pour la production de bois doit combiner cinq lignes d'actions publiques :

- un mécanisme public d'assistance technique forestière, qui consolide la gestion conjointe d'activités agricoles et forestières au sein des exploitations ;

- des actions de support visant la consolidation des organisations paysannes gestionnaires d'aménagement forestier ; - un programme de crédit spécialisé visant l'implantation des plans d'aménagement paysans ;

- une amélioration des procédures administratives d'autorisation d'exploitation ;

- des règles et des normes établies visant un plus grand contrôle des organisations paysannes sur la commercialisation du bois.

\section{L’assistance technique forestière}

Il existe déjà au niveau des États des organismes d'assistance technique publique pour la production agricole paysanne amazonienne. Malgré leurs limites, celles d'institutions créées dans le cadre de la Révolution verte des années 1970, ils représentent une offre publique d'assistance technique avec des bureaux répartis dans les régions, et une capacité d'élaboration de projets de crédits individuels ou collectifs. Dans le Pará et l'Amazonas, ces organismes intègrent progressivement des compétences sur le thème forestier, avec l'appui du Sfb.

Ces organismes restent limités, s'agissant notamment du choix des modèles productifs à promouvoir dans le domaine paysan, et des types d'assistance technique à mettre en place.

Le cadre général de la législation rurale évolue en Amazonie, ce qui ne facilite pas les choix stratégiques en matière de modèles productifs. Le nouveau code forestier, en phase finale d'approbation au niveau fédéral en 2012, doit notamment diminuer de $80 \%$ à $50 \%$ la part de réserve forestière obligatoire (Arl) dans certaines régions, et préciser les alternatives productives autorisées pour sa récupération (forêts plantées, systèmes agroforestiers ou sylvopastoraux, possibilité d'inclusion d'espèces exotiques au milieu, etc.).

Cependant, la tendance est au renforcement de la composante forêt des systèmes de production et doit mener à la mise en place de systèmes mixtes agriculture-forêt, avec des caractéristiques différentes selon les contextes régionaux et fonciers : aires protégées, périmètres de colonisation individuelle ou collective, terres privées. L'effort pour développer la compétence forestière dans ces organismes doit donc être nettement accéléré : embauche d'ingénieurs et techniciens forestiers, formation. Le conseil en gestion des exploitations doit aussi évoluer de façon à intégrer les aspects de gestion patrimoniale et financière à long terme, spécifiques de la foresterie. Les organisations pay- 
sannes elles-mêmes doivent être appuyées, de par la complexité technique, administrative, juridique et financière de la gestion des aménagements durables des forêts.

Ainsi le type d'assistance technique à offrir doit-il évoluer, si on le compare au système classique de la vulgarisation rurale. D’autres fonctions doivent être développées, entre autres :

- une programmation de formation technique à moyen/long terme destinée aux populations paysannes, différente des classiques sessions ponctuelles, courtes et spécifiques ; il est possible de comparer cette nécessité au travail réalisé par la Ceplac pour diffuser la production commerciale de cacao parmi une paysannerie amazonienne qui ne connaissait que très peu les techniques d'arboriculture ;

- un travail spécifique sur les organisations paysannes détentrices juridiques des plans, qui doit concerner la gestion de l'aménagement (technique, comptable et financière, gestion de contrats, gestion de la commercialisation, gestion des partenariats). C'est ce que fait classiquement le Sebrae dans l'appui aux Pme, ou dans les pépinières d'entreprises.

\section{Le financement de l’aménagement forestier paysan}

Le financement peut être une stratégie pour changer le cadre financier de la relation des communautés paysannes avec les entreprises, avec des effets sur la durabilité et la gouvernance de la gestion de l'aménagement.

Les activités d'aménagement peuvent être séparées en deux étapes principales : la phase pré-exploitation, qui se conclut par l'autorisation administrative d'exploiter, et la phase d'exploitation. Dans le cas de l'Acoprasa, le contrat avec l'entreprise recouvre les deux étapes : l'entreprise exécute l'ensemble des opérations d'aménagement au nom de l'association, dans une relation proche d'un contrat de fermage. Dans le cas de la Coomflona, la première étape est exclusivement accomplie par les paysans, et le document administratif décrivant la volumétrie exploitable par espèce permet de signer les contrats de commercialisation avant l'exploitation. La coopérative peut ainsi négocier l'anticipation d'une part du paiement, et faciliter le financement de la phase d'exploitation. Ses associés conservent ainsi une grande autonomie pour les principales décisions de gestion de l'aménagement (surface de coupe annuelle, espèces à exploiter), et peuvent dimensionner au mieux la participation des entreprises dans la phase d'exploitation.

Du point de vue des politiques publiques, ce résultat est important : la phase pré-exploitation est donc stratégique pour pouvoir contrôler les conditions de commercialisation, ce qui impacte fortement les résultats économiques de l'aménagement dès que l'on a résolu les questions de formation technique.
Les coûts de la phase pré-exploitation ont été estimés à $13-20 \%$ des coûts totaux de l'aménagement, soit 123 200 R\$/ha exploités dans le cas de la Coomflona et de l'Acoprasa. Ainsi, un crédit de 12000 R\$ par famille, inférieur au volume actuellement distribué par colon par l'Incra, est suffisant pour financer la phase pré-exploitation pour une famille disposant de 60 ha de forêt à aménager.

Il s'agit donc de structurer un programme de crédit spécifique, collectif ou familial, dans le cadre des aires protégées ou des périmètres de colonisation, visant le financement de la phase pré-exploitation des plans d'aménagement paysans. Il est possible de mobiliser pour ce faire des ressources existantes et non utilisées, comme le programme Pronaf Florestal du ministère du Développement agraire.

\section{Conclusion}

Les expériences analysées montrent d'abord que la production forestière paysanne est viable dans le cadre actuel des politiques publiques en Amazonie. Cependant, pour développer cette production vers un volume compatible avec les nécessités du secteur forestier du Pará, une politique publique doit être mise en œuvre, en complément à la loi de gestion durable des forêts publiques de 2006, pour laquelle la présente étude a proposé et analysé des pistes prometteuses.

\section{Remerciements}

Cette étude a été réalisée dans le cadre du projet Floresta em Pé financé par le Ffem et le gouvernement brésilien de 2007 à 2011. Les auteurs tiennent à remercier tout particulièrement tout le personnel de la COOMFLONA, de la MAFLOPS et d'ACOPRASA pour leur collaboration, la mise à disposition des données d'exploitation.

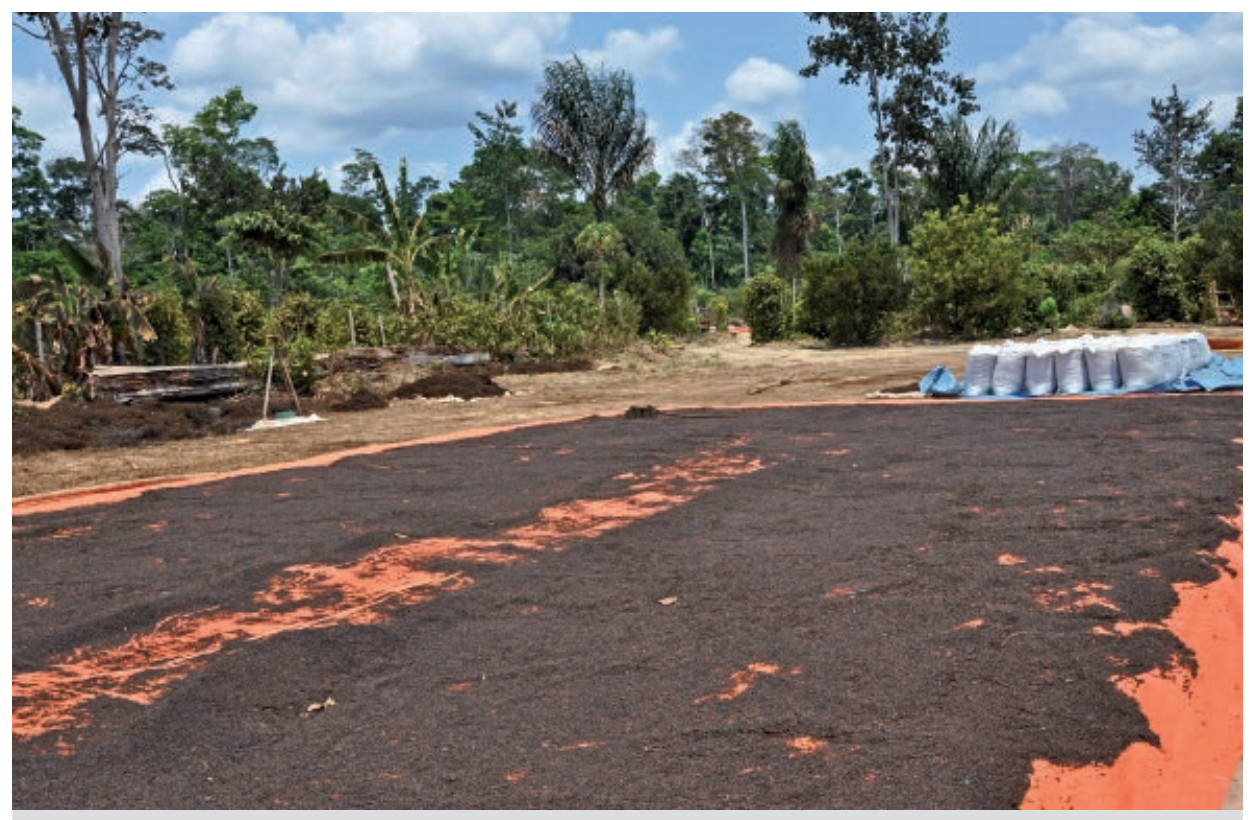

Séchage du poivre et empaquetage dans une propriété d'un agriculteur de la communauté de Santo Antonio. Photo P. Sist. 


\section{Références bibliographiques}

BARTHELON S., 2009. Diagnóstico Agro Econômico das Vicinais km 124 e 145 BR 163 (Santarém - Cuiabá), estado do Pará Brasil. Rapport de fin d'études, AgroParistech, Paris, 121 p.

CNS, IDESAM, 2010. Seminário Governança Florestal no Amazonas: cenários para a Consolidação do Manejo Florestal no Estado, destaques do Seminário, 18-19/11/2010. Manaus-AM, Brésil, auditório da Escola Superior de Tecnologia EST/UE, 35 p. http://api.ning.com/files/n3Z0tr Jyv5eajZ5TWIZ1rYF73fV5dg8WJdWLkMmS3uLToaFMSHywl NuhlknrR10tgfYNPXE1RjBbUb7KJdT766BT9fpywWcB/Desta quesSeminario.pdf

CRUZ H., SABLAYROLLES P., KANASHIRO M., AMARAL NETO M., SIST P. (ORG.), 2011. Relação empresa/comunidade no contexto do manejo florestal comunitário e familiar: uma contribuição do projeto Floresta em Pé. Belém-PA, Brésil, Ibama/Dbflo, 318 p. http://www.gret.org/publication/relacao-empresacomunidade-no-contexto-do-manejo-fl-orestalcomunitario-e-familiar-uma-contribuicao-do-projeto-floresta-em-pe-relacao-empresacomunidade-no-contexto-domanejo-fl-orestal-comunitario-e/

DRIGO I. G., PIKETTY M.-G., PANTOJA PENA J. W., 2009. CUStos e benefícios da implementação de planos de manejo florestal comunitário na região da Transamazônica (Pará). Belém-PA, Brésil, Projeto Floagri, 61 p.

DUFUMIER M., 1996. Les projets de développement agricole. Manuel d'expertise. Paris, France, Karthala, Wageningen, Pays-Bas, CTA, 354 p.

IDEFLOR, 2009. Minuta do Plano de Outorga Florestal 2010. Belém-PA, Brésil, Governo do Pará, Ideflor, 93 p.

ISA, IMAZON, 2011. Áreas Protegidas na Amazônia brasileira: avanços e desafios. Belém-PA, Brésil, Imazon, São Paulo-SP, Brésil, Isa, 90 p. http://www.imazon.org.br/publicacoes/ livros/areas-protegidas-na-amazonia-brasileira-avancos-e

MONTEIRO A., CARDOSO D., CONRADO D., VERÍSSIMO A., SOUZA Jr. C., 2011. Transparência Manejo Florestal, Estado do Pará, 2009 a 2010. Belém-PA, Brésil, Imazon, 16 p. http://www.imazon.org.br/publicacoes/transparenciamanejo-florestal/transparencia-manejo-florestal-do-estadodo-para-2009-e-2010

PEREIRA D., SANTOS D., VEDOVETO M., GUIMARÃES J., VERÍSSIMO A., 2010. Fatos florestais da Amazônia 2010. Belém-PA, Brésil, Imazon, 124 p. http://www.imazon.org. $\mathrm{br} /$ publicacoes/livros/fatos-florestais-da-amazonia-2010)

PINTO A., AMARAL P., AMARAL NETO M., 2011. Iniciativas de manejo florestal comunitário e familiar na Amazônia brasileira, 2009/10. Brasília-DF, Brésil, Imazon, leb, Belém-PA, Giz, Sfb, 86 p. http://www.sfb.gov.br/publicacoes/tecnicocientifico/iniciativas-de-manejo-florestal-comunitario-efamiliar-na-amazonia-brasileira-2009/2010

SFB, IMAZON, 2010. A atividade madeireira na Amazônia brasileira: produção, receita e mercados. Belém-PA, Brésil, Sfb, Imazon, 20 p. http://www.imazon.org.br/ publicacoes/livretos/a-atividade-madeireira-na-amazonia-brasileira
SFB, 2007. Manejo florestal comunitário na Amazônia brasileira: avanços e perspectivas para a conservação florestal. Brasília-DF, Brésil, Ministério do Meio Ambiente, Sfb, 21 p. http://www.sfb.gov.br/publicacoes/apoio-didatico/manejoflorestal-comunitario-na-amazonia-brasileira

SFB, 2009. Plano de Outorga Florestal 2010. Brasília-DF, Brésil, Ministério do Meio Ambiente, Sfb, 101 p. http://www.sfb.gov.br/concessoes-florestais/processo-deconcessao/paof-2010

SFB, 2011. Plano anual de outorga florestal 2012. Brasília-DF, Brésil, Ministério do Meio Ambiente, Sfb, 130 p. http://www.florestal.gov.br/publicacoes/instrumento-de-gestao

SFB, 2012. Pré-edital de licitação Flona do Amana - Lote II, Anexo 1: Relação das Unidades de Manejo Florestal. Brasília-DF, Brésil, Ministério do Meio Ambiente, Sfb. http://www.florestal.gov.br/pre-editais-lancados/

SIST P., NASCIMENTO-FERREIRA F., 2007. Sustainability of reduced-impact logging in the Eastern Amazon. Forest Ecology and Management, 243: 199-209. 